STRUCTURAL SCIENCE CRYSTAL ENGINEERING MATERIALS

ISSN 2052-5206

\section{Plasmonic Effects in Metal-Semiconductor Nano- structures. By Alexey A. Toropov and Tatiana V. Shubina. Oxford University Press, 2015. Pp. 384. Price GBP 75.00. ISBN 9780199699315.}

\author{
Michel Vergnat*
}

Université de Lorraine, Faculté des Sciences et Technologies, Institut Jean Lamour UMR CNRS 7198, BP 70239, 54506 Vandoeuvre-lès-Nancy CEDEX, France. *Correspondence e-mail: michel.vergnat@univ-lorraine.fr

Plasmonics is a field of study that explores the interaction of light waves and metallic surfaces, and the resulting electron density waves that can be generated from this interaction. The resulting electron density wave that propagates along the surface of the metal is referred to as a surface plasmon polariton, or a surface plasmon. Surface plasmons present many applications. They can be used to control colours of materials, as illustrated by the historic stained glass of medieval cathedrals. In this case, the colour is given by metal nanoparticles which interact with light. Surface plasmons also play a role in Wood's anomalies in light reflection from a metal diffraction grating, observed in 1902, or in surface-enhanced Raman scattering, observed in 1974. Later, the field of plasmonics exploded when it was shown that tiny metallic structures can enable optical circuitry at the nanoscale, and that an array of nanoscale holes in a thin metal film can exhibit extraordinary optical transmission.

New exciting pathways to control light in ultra-compact geometries have been opened by implementation of active plasmonic devices in addition to passive metallic lightconcentration structures. Among these recent achievements are plasmonic nanolasers or spasers, as well as plasmon-enhanced light-emitting diodes, detectors, solar cells and single quantum emitters. All these applications rely on the effects of light-matter coupling in nanostructures, including semiconductors and metals, or other conducting materials such as degenerate semiconductors, semimetals or graphene. Furthermore, strong coupling between surface plasmons and excitons has been observed in plasmonic cavity structures, including both organic and inorganic semiconductors.

The book Plasmonic effects in metal-semiconductor nanostructures focuses on the performance of plasmonics related to semiconductor metal nanostructures. The purpose is to give a general view of electromagnetic and quantum phenomena emerging in metalsemiconductor plasmonic structures, ranging from basic physical theory to the practical engineering applications such as single-photons sources, nanolasers, ultra-compact modulators, and so on. After a first chapter which describes the milestones of plasmonics and the scope of the book, the content is divided into three parts. The first part (fundamentals) starts with the foundations of metal-semiconductor plasmonics. It contains general information on plasmonic effects in metal structures, as well as electronic and optical properties of semiconductor structures, acting as building blocks of the devices of active plasmonics. The second part (materials) describes characteristics of particular materials, both conducting and semiconducting, which can be of value for the design of hybrid plasmonic structures. The third part (metal-semiconductor nanostructures) describes the existing theoretical approaches to the description of light-matter coupling in metal-semiconductor structures and presents proof-of-concept experimental observations. This part is essentially devoted to the practical applications of active plasmonics.

Chapter 2 (electrodynamics of metal structures) outlines basic ideas and definitions of classical electrodynamics, focusing on the propagation of plane waves, the boundary conditions between two media, and the derivation of the dielectric function of the free electron gas in the framework of the Lorentz oscillator model and the Drude theory. A detailed presentation of the various types of plasmons is given, including the bulk 
plasmon polaritons, the surface plasmon polaritons (SPPs) propagating at metal-dielectric interfaces, and the localized surface plasmons (LSPs) in metal particles. In the latter case, both a quasistatic approach and the exact Mie theory are considered.

Chapter 3 (electronic excitations in semiconductors) covers the topic of electronic band structures in semiconductor materials, which are the most important materials in today's electronic and optoelectronic technologies. Relevant concepts are introduced including Bloch's theorem, symmetry theory based on the mathematical theory of group representations, the k.p. theory which permits the analytical expressions for band dispersion to be simply obtained around high-symmetry points. This description is focused on direct band-gap semiconductors with zinc-blende and wurtzite crystal structures [(III)-(V) and (II)-(VI) compounds], which present efficient photoluminescence. Indirect band-gap semiconductors such as silicon and germanium are not discussed in this book. Kane and Luttinger-Kohn models are presented for the zinc-blende crystal structure, while the electronic band structure for the wurtzite-type semiconductors is defined within the model developed by Rashba, Sheka and Pikus. Effects of elastic strain on the band structures are also described.

Chapter 4 (optics in semiconductors) considers basic optics in semiconductor crystals, in particular the optical absorption, gain and emission. The concept of density of states is introduced for systems of different dimensionality. The interband absorption and emission processes in semiconductors are described within a semiclassical approach. The Einstein coefficients are introduced in order to define the connection between absorption, stimulated emission and spontaneous emission. Expressions for the interband transition matrix elements, i.e. the optical selection rules, are presented in terms of the k.p. theory. The concept of Wannier-Mott excitons in semiconductors are discussed in the framework of the effective mass approximation, providing symmetry classification of excitonic states in the semiconductor crystals.

Chapter 5 (conducting materials) covers available conducting materials capable of supporting plasmonic excitations. Noble (gold, silver) and polyvalent (indium, aluminium) metals, degenerate semiconductors, doped oxides and semimetals, as well as graphene are all described here. Indeed, at optical and near-IR frequencies, most studies of plasmonic effects have been performed using metals. However, in degenerate semiconductors and graphene, the density of free carriers can be so high that they start to act in the far-IR and terahertz ranges. Transparent conducting oxides, such as metal-doped $\mathrm{ZnO}$ and indium-tin oxide, as well as semimetals (silicides and germanides) also have applications for near-IR plasmonics and plasmonic photovoltaics. Where possible, the explicit expressions and the plots defining the spectra of complex dielectric functions and other important characteristics are given.

Chapter 6 (semiconductor materials and nanostructures) introduces the physics of semiconductor heterostructures and nanostructures. With the development of sophisticated growth techniques, it is now possible to fabricate nanometer-scale semiconductor structures which are of great interest since their properties are modified as a result of reduced dimensionality. The chapter presents the electronic and optical properties of the most common III-V and II-VI binary semiconductor compounds and their alloys. The concept of pseudomorphic heterostructures made from strained layers of these materials is defined. The basic effects of spatial confinement of carriers on the electronic and optical properties are considered, focusing on two-dimensional structures (quantum wells) and zero-dimensional structures (quantum dots). In both cases, the electron spectra and optical transitions are defined in the framework of a multiband envelope function approximation. The fine structures of excitons (both in quantum wells and quantum dots) are represented in terms of symmetry taking into account both the symmetry of the materials with either zinc blende or wurtzite structures and the symmetry reduction in the whole heterostructure.

Chapter 7 (light-matter interactions in metal-semiconductor structures) defines electromagnetic effects inducing magnification of optical absorption and optical emission in a semiconductor due to enhancement of both the optical field and the density of electromagnetic modes in the vicinity of a metal object. Indeed, the metal object increases the optical energy density that falls on the semiconductor structure and thereby increases the absorbed optical power. Another contribution to the electromagnetic enhancement originates from an increase in the spontaneous emission rate. This mechanism relies on the increasing density of photonic modes and is known as the Purcell effect. Some practical calculations are presented, describing the manifestations of the electromagnetic enhancement in simplified model structures.

Chapter 8 (frontiers in plasmonics applications) contains seven sections which cover a wide range of promising applications based on the effects of coupling plasmonic excitations to distinct optical resonances in hybrid structures combining conductors and semiconductors. The discussions rely on the results obtained in all previous chapters. $\S 1$ is devoted to the phenomenon of surface-plasmon-enhanced emission in quantum wells and quantum dots, which are promising as active regions of light-emitting diodes. The strong coupling regime in plasmonic cavities is presented. The applications include the plasmonic nanolasers and antennas. $\$ 2$ shows that the coupling, with a plasmonic cavity, of emitters such as single semiconductor quantum dots or single impurities in semiconductor crystals can facilitate the enhancement of narrow emission lines related to single excitons and improve their photostability. $\S 3$, which describes the spin-polarized plasmonics, includes a presentation of the optical spin Hall effect and the traffic of polarized light. $\S 4$ is concerned with the quantum information technology, the single-photon emitters and the creation of entangled photon pairs. $\S 5$ focuses on the properties of nanocomposites which contain metallic nanoclusters inside an optically active semiconductor host. Their applications in photovoltaics are highlighted. The problems of IR and terahertz plasmonics based on doped semiconductor nanostructures as well as properties of systems 
with an index near zero are reviewed in $\$ 6$ and plasmonic applications of graphene are outlined in $\S 7$.

In summary, this book is divided into three parts. The first part clearly recalls the foundations of physics useful for understanding the plasmonic effects in metal-semiconductor nanostructures. The second part plainly describes the electronic properties of conducting materials, of III-V and II-VI semiconductors, and of nanostructures. The third part is dedicated to applications. The first two sections will be very useful to researchers who want to discover the area of plas- monics related to semiconductor-metal nanostructures. These parts could be readable by good graduate students. The third part is more specialized. It gives a very detailed review of the applications of the metal-semiconductor nanostructures, with numerous references (the references of the book are listed through 37 pages). The 122 tables and figures are clearly presented. As a conclusion, I recommend this book which can be very useful to researchers in physics, optical engineering and material science who are interested in the field of nanophotonics. 